\title{
LOW VOLTAGE PIEZOELECTRIC NEMS RELAYS FOR ULTRALOW POWER DIGITAL CIRCUITS
}

\author{
Usama Zaghloul ${ }^{1,2} *$ and Gianluca Piazza ${ }^{l}$ \\ ${ }^{1}$ Electrical and Computer Engineering Department, Carnegie Mellon University, Pittsburgh, Pennsylvania, USA \\ ${ }^{2}$ Microelectronics Department, Electronics Research Institute, Cairo, Egypt
}

\begin{abstract}
The paper presents the design, synthesis, and characterization of the first piezoelectric nanoelectromechanical relays with sub-1Volt actuation $(850 \mathrm{mV})$. The relays are normally closed and exhibit high mechanical restoring forces at relatively low voltage. This is enabled by (i) employing ultrathin piezoelectric aluminum nitride film $(10 \mathrm{~nm})$ with high $c$-axis orientation and controlled residual stress, (ii) demonstrating a relay design with a stresscompensating geometry, and (iii) optimizing the thicknesses of the layers forming a four-layer unimorph actuator. The relays exhibit a very low energy dissipation per switching cycle (46 aJ), an extremely small subthreshold slope $(0.015 \mathrm{mV} / \mathrm{dec})$, and a low contact resistance $(16 \mathrm{k} \Omega)$.
\end{abstract}

\section{INTRODUCTION}

NEMS relays have been proposed as very promising successors or hybrid complements to metal-oxide-semiconductor field-effect transistors (MOSFETs) [1]. The fundamental physics of MOSFETs limit their further scaling due to the sharp increase in static power consumption and the inability to lower the supply voltage below few 100s of milliVolts [2]. NEMS relays, however, have been demonstrated to implement digital logic [3, 4] and memory [5-6] elements with low leakage and extremely steep subthreshold slope. They are also more suitable for harsh environments compared to MOSFETs [7]. Despite these merits, the high voltage presently used to drive NEMS relays $(2.5-22 \mathrm{~V})$ stands against their use in ultralow-power applications. Although electrostatic NEMS relays were recently demonstrated to operate at $0.4 \mathrm{~V}$ [8], piezoelectric relays with sub-1-Volt actuation have never been reported. Two main challenges stand against achieving low actuation voltage in miniaturized piezoelectric relays: (i) synthesizing ultrathin piezoelectric films with high quality, and (ii) controlling the stress-induced deflection in the multi-layer stack of piezoelectric actuators to maintain few nanometers height switching gaps. An actuation voltage smaller than $1 \mathrm{~V}$ could not be achieved even for aggressively scaled piezoelectric NEMS relays which use ultrathin $(25 \mathrm{~nm})$ AlN films and $\sim 28 \mathrm{~nm}$ switching gaps [9].

This work reports the design and fabrication of normally closed (NC) piezoelectric NEMS relays which exhibit an actuation voltage smaller than $1 \mathrm{~V}$. The relays are formed by the thinnest to date piezoelectric AlN films $(10 \mathrm{~nm})$ and use a stresscompensating geometry and an optimized unimorph actuator to maximize the restoring mechanical forces.

\section{RELAY DESIGN AND OPERATION}

The NC relay has four terminals: source, drain, gate, and body (see Fig. 1), and is based on piezoelectric unimorph actuators. The relay makes use of a stress-compensating geometry, and consists of three suspended beams placed next to each other (Fig. 1a and b). The center beam carries the piezoelectric actuator and the metal tip (the conductive channel) in the longitudinal direction of the beams while the two outer symmetric beams carry the source and drain bottom contacting metals in the transverse direction. The outer beams are connected together by an insulating layer of AlN, hence forming a folded beam structure surrounding the center actuator

(see Fig. 1c). We refer to this arrangement as a triple-beam structure. From a stress perspective, the triple beam geometry resembles a differential topology. The residual stress and stress gradient of the center beam are an average of the stresses of the outer beams, independently of whether the stresses of the outer beams are equal or different. Consequently, the stress-induced deflection of the center beam is an average of the deflections of the outer beams. This compensates the differences in stresses between the three beams and maintains the nanometer height switching gaps. Note that the relay geometry makes use of curved beams which average the in-plane stresses in $x$ and $y$ directions simultaneously between the center and outer beams across the whole beams' length. This in turn reduces further the displacement difference due to any stress asymmetry in the three beams. Rectangular beams, however, would compensate only the stress in either $x$ or $y$ directions. FEA simulations were performed for the proposed triple beam structure under different residual stresses using COMSOL. Fig. 1b shows an example of these results where the left, center and right beams with respective residual stresses of 230, 140 and $35 \mathrm{MPa}$ exhibit comparable deflections.

(a)
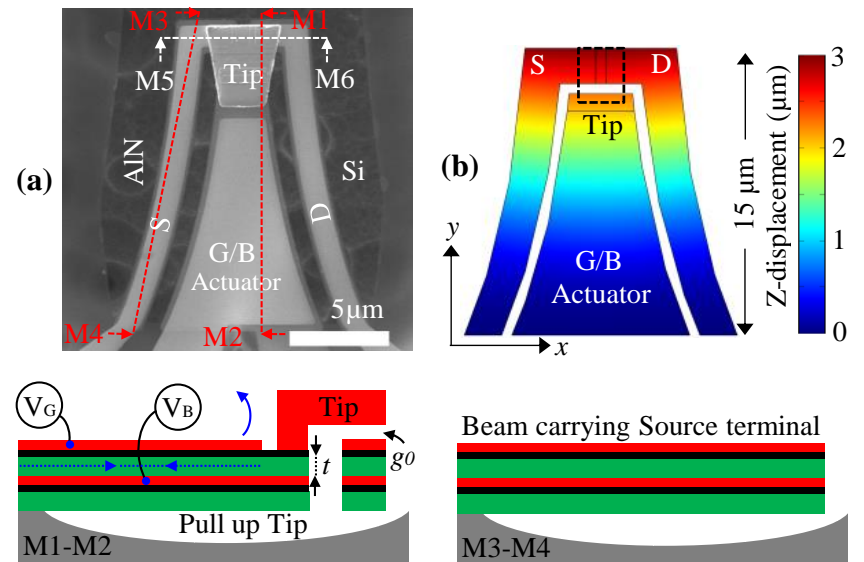

go: switching gap (=0 $\mathrm{nm}$ for $\mathrm{NC}$ relays)

$t$ : AlN piezoelectric film thickness

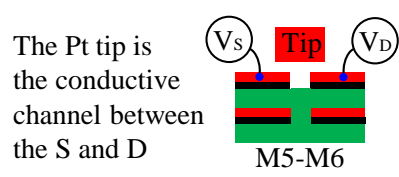

(c)

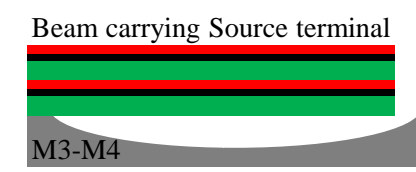

Source: $\mathrm{S}$

Drain: D Gate: $\mathrm{G}$ Body: B

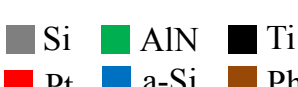
Photoresist (c)

Figure 1: NC NEMS relays based on the triple-beam geometry: (a) $S E M$ images of as fabricated piezoelectric NC relay using $10 \mathrm{~nm}$ thick AlN films; (b) FEA simulation showing the stress-induced deflections for the three suspended beams; (c) cross sections describing the layer stack of the beams and the relay structure.

In NC relays, the tip contacts the source and drain upon releasing the device without any applied voltage (i.e. the relay is normally on as shown in Fig. 2). In order to achieve that, the residual stresses in the layers forming the beams were reduced, yet kept large enough to yield a higher divergence in the stress levels 
of the three beams than the stress margin that can be compensated by the triple beam geometry. This margin depends also on the initial switching gap determined by the thickness of the sacrificial layer. For the switches realized in this work, the residual stresses of the AlN and Pt films were -250 and $\pm 75 \mathrm{MPa}$, respectively and the sacrificial layer thickness was $60 \mathrm{~nm}$. To turn off the relay, a small restoring force is required to overcome only the stress asymmetry in the three beams (in addition to surface adhesion), hence a low actuation voltage is expected.

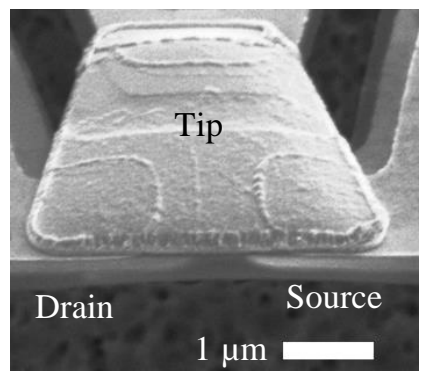

Figure 2: Magnified SEM image of the contact area for as fabricated NC relay. The tip contacts the source and drain upon releasing the device due to asymmetric in-plane stresses.

We performed a FEA simulation to determine the stress-induced zdeflections for the three beams forming the relay shown in Fig. 1a. The analysis made use of the measured residual stress values for AlN and Pt (mentioned above), and assumed a 20\% variance in the stress levels between the three beams. An initial gap of $60 \mathrm{~nm}$ (the sacrificial layer thickness) was included between the metal tip and the source and drain contacting metals. Also, no contact pair was defined between the tip and the source and drain in order to determine the deflection of the center beam at the contact area. The simulation shows that the z-deflections at the contact area for the left, center and right beams are 2.079, 1.986 and $2.035 \mu \mathrm{m}$, respectively (see Fig. 3). In other words, the z-position of the metal tip is 93 and $49 \mathrm{~nm}$ below the source (left beam) and drain (right beam), respectively, hence forming a NC relay. The simulation highlights also that if the residual stress of $\mathrm{AlN}$ and $\mathrm{Pt}$ is reduced to $-50 \mathrm{MPa}$, and the stress variation between the three beams is decreased to $5 \%$, a normally open (NO) relay with $8 \mathrm{~nm}$ switching gap can be obtained.

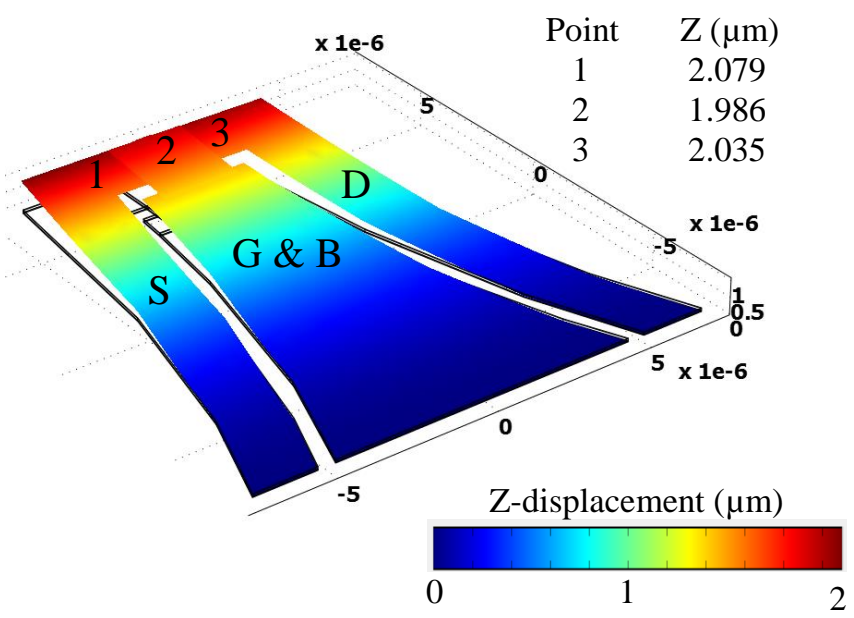

Figure 3: FEA simulation for the residual stress-induced deflections in the synthesized $N C$ relays. The measured residual stresses for the sputtered AlN and Pt films were used in simulation.
The NC relay demonstrated in this study uses a four-layer piezoelectric unimorph actuator (see Fig. 4a). The actuator consists of a piezoelectric film (AlN) sandwiched between two metal electrodes (the gate and body), and bonded to an elastic layer (AlN). Applying an electric field between the body and gate terminals generates a lateral strain in the piezoelectric film. Due to the constraint imposed by the elastic layer, the lateral strain is translated to a transverse moment around the neutral axis of the actuator's stack causing a bending motion. The direction of the electric field across the piezoelectric film determines the bending direction of the actuator. In the synthesized NC relay, the actuator bends in the upward direction upon applying the actuation voltage, which pulls the tip (the conductive channel) far from the source and drain, hence turning the relay off (Fig. 4a). Removing the applied voltage causes the actuator to return to its original position, shortening the source and drain. Thus, the gate-body potential difference is used to create or remove a conducting channel between the source and drain via a metal tip.
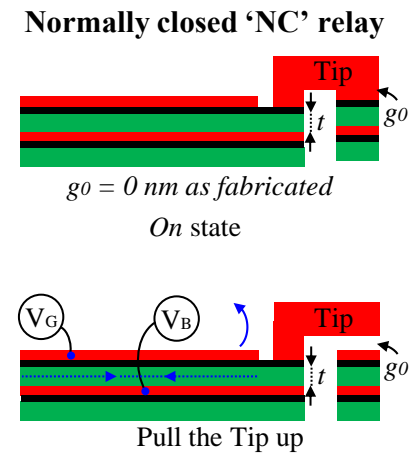

Off state

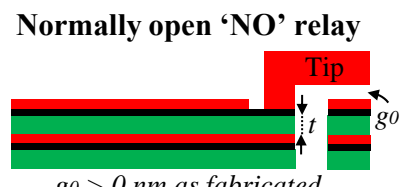

go $>0 \mathrm{~nm}$ as fabricated Off state

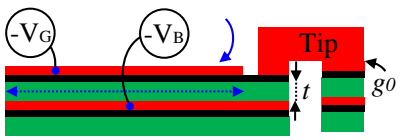

Push the Tip down

On state (a)

(b)

Figure 4: Cartoon schematic describing the operating principle of piezoelectric (a) NC and (b) NO relays.

The thicknesses of the elastic layer (the bottom AlN) and the $\mathrm{Ti} / \mathrm{Pt}$ electrodes forming the piezoelectric unimorph actuator were optimized to maximize the electromechanical coupling. As a result, the synthesized NC relay exhibits high mechanical restoring force at relatively small voltage. Therefore, a low actuation voltage can be achieved. This analysis was performed using FEA simulation and a complex analytical model that considers all layers in the actuator stack [10]. Here it is worth mentioning that the optimization goal of the unimorph actuators used to realize $\mathrm{NC}$ and NO relays is different. In NC relays, the initial switching gap, $g_{0}$, is $0 \mathrm{~nm}$ (i.e. the relay is in the on state as fabricated as highlighted in Fig. 4a). The thicknesses of the layers forming the actuator are optimized to result in a maximum restoring force in order to break the contact during the opening of the relay at small voltages. In a NO operation (Fig. 4b), the initial $g_{0}$ is larger than $0 \mathrm{~nm}$, and the actuator has to close this gap to turn the relay on. In this case, the thicknesses of the actuator's layers are selected as a compromise between generating maximum displacement and maximum contact force. This is required to assure that the relay can be turned on using a small voltage while exhibiting a low contact resistance.

\section{FABRICATION PROCESS FLOW}

The relays were fabricated using a seven masks process as described in Fig. 5. The most critical and new step in the process with respect to what was reported in [9] was the reactive sputtering of highly $c$-axis oriented $10 \mathrm{~nm}$ thick AlN films with controlled 
residual stress over ultrathin Pt layers $(5 \mathrm{~nm})$. A two-step deposition recipe was developed to sputter AlN using a dual cathode S-Gun magnetron source. Each step exhibited a different process work point on the hysteresis curve of the magnetron discharge via changing the $\mathrm{N}_{2}$ and Ar partial pressures to provide better conditions for AlN nucleation on the ultrathin Pt surface. The in-plane stress of the AlN films was controlled via modifying the Ar flow gradually during the reactive sputtering process.
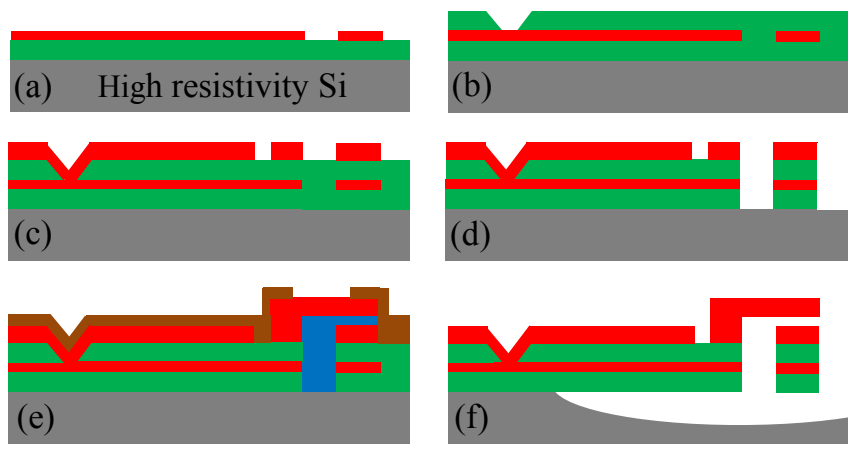

$\mathrm{Si} \quad \mathrm{AlN} \square \mathrm{Ti} / \mathrm{Pt}$

Cross-sectional high-resolution transmission electron microscopy (HRTEM) image of the four-layer piezoelectric actuator used to realize the NC relays is shown in Fig. 6. The image highlights the high $c$-axis orientation and the fine columnar grain structure of the synthesized $10 \mathrm{~nm}$ AlN films. This can be observed for the piezoelectric film (top AlN) as well as the elastic layer (bottom AlN). Moreover, the arrangement of the lattice planes in the grown ultrathin AlN film can be seen, and emphasize the continuous lattice microstructure within a single grain from the AlN/Pt interface up to the film surface. The full width at half maximum (FWHM) of $\mathrm{x}$-ray diffraction rocking curve measurements around the diffraction peak of AlN (0002) for the sputtered $10 \mathrm{~nm}$ films is $4.7^{\circ}$. Also, the deflection profiles of the synthesized four-layer unimorph actuators were measured under different applied voltages using an optical profilometer. These measurements were fitted to the computed deflections from the analytical model in [10] in order to calculate the $d_{31}$ piezoelectric coefficients for the sputtered piezoelectric films. The extracted $d_{31}$ coefficient for the synthesized $10 \mathrm{~nm}$ AlN films is $-1.75 \mathrm{pC} / \mathrm{N}$, which is $91 \%$ of the reported $d_{31}$ value for $1 \mu \mathrm{m}$ thick and highly oriented AlN films. This clearly highlights the significance of the developed two-step recipe for the reactive sputtering process.

Figure 5: The fabrication process described at the cross section M1-M2 shown in Fig 1a: (a) Sputter $10 \mathrm{~nm}$ AlN seed layer on (100) Si substrate, sputter and pattern Ti/Pt (2/5 nm) layers using lift-off to define the actuator's bottom electrode; (b) deposit $10 \mathrm{~nm}$ piezoelectric AlN film using reactive sputtering, open vias (not shown) in the AlN using the AZ400K developer to access the bottom electrode; (c) deposit and pattern Ti/Pt layers to define the actuator's top electrode and the source and drain; (d) define the lateral dimensions of the relay by AlN etching using $\mathrm{Cl}_{2} / \mathrm{BCl}_{3} / \mathrm{Ar}$ chemistry; (e) sputter and pattern a sacrificial layer of amorphous Si $(60 \mathrm{~nm})$ by lift-off, deposit and pattern a Pt layer via lift-off to define the tip and contact pads, define release openings using a photoresist mask; ( $f$ ) etch the sacrificial layer and the Si substrate using $\mathrm{XeF}_{2}$ to release the relay and remove the photoresist by plasma ashing.

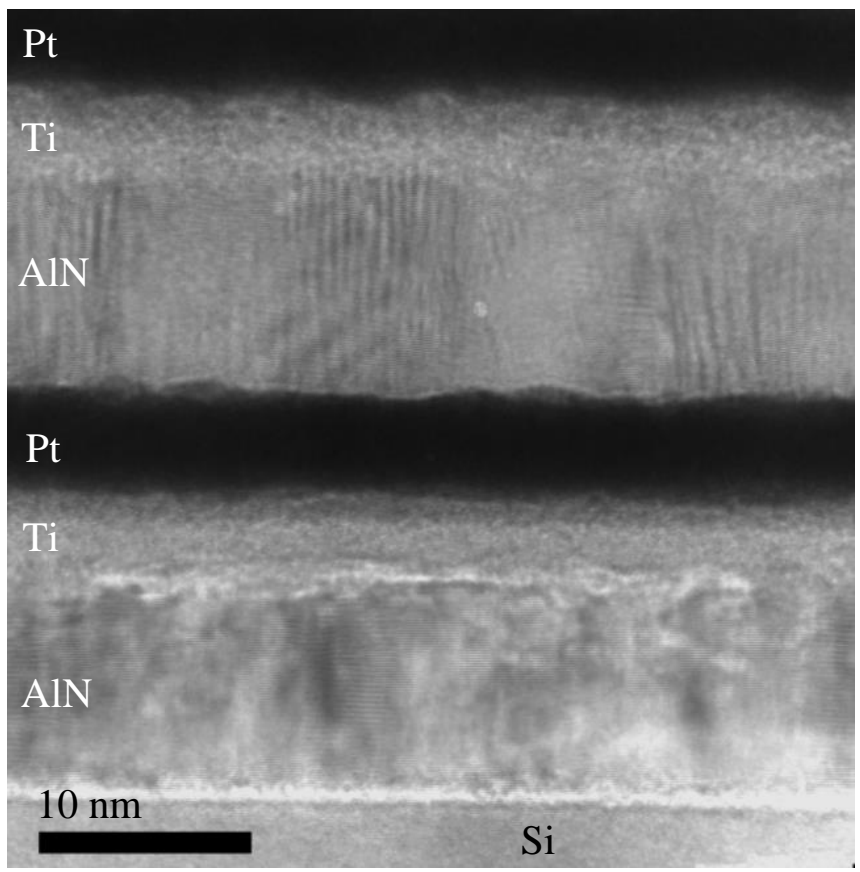

Figure 6: HRTEM image showing the layer stack of the piezoelectric unimorph actuator used to realize the NC relays.

\section{EXPERIMENTAL RESULTS AND DISCUSSION}

Figure 7a presents typical I-V measurements for the synthesized NC relays based on $10 \mathrm{~nm}$ thick piezoelectric AlN films. The measurements were done in ambient air using the bodybiased actuation method [11]. The shown turn-off voltage sweeps were recorded while the body terminal was biased using different voltages. The role of the bias applied to the body, $V_{B}$, is to increase the mechanical restoring force of the actuator (see Fig. 1 and 4a) so that a small complementary voltage applied to the gate terminal $\left(V_{G}\right)$ can break the contact and turn the relay off. The gate voltage at which the relay is turned off is called the threshold voltage, $V_{t h}$. As shown in the figure, $V_{t h}$ can be precisely tuned and reduced to few millivolts via adjusting $V_{B}$. The relay exhibits an actuation voltage $\left(V_{a c t}=V_{t h}-V_{B}\right)$ of $850 \mathrm{mV}$ and a source-drain resistance of $16 \mathrm{k} \Omega$ (Pt-to-Pt contact).

The average $V_{t h}$ measured under different $V_{B}$ for the demonstrated NC relays is plotted in Fig. 7b. The linear dependence of $V_{t h}$ on $V_{B}$ is obvious. For comparison, the measurements of a NO relay which uses $25 \mathrm{~nm}$ thick piezoelectric AlN film and $28 \mathrm{~nm}$ switching gap is also plotted in the figure [9]. Thanks to the scaling of the AlN film thickness to $10 \mathrm{~nm}$ and the proposed NC design demonstrated in this work, the relays exhibit an actuation voltage smaller than $1 \mathrm{~V}$ compared to $1.6 \mathrm{~V}$ for the NO relays. A comparison between the synthesized NC relays in this study and prior work on NO AlN relays is summarized in Table 1. The sub-1-Volt actuation of the NC switch designated as $\mathrm{S} 1$ comes with more than $3 \mathrm{X}$ reduction in the relay active area compared to S2, the NO relay that used $25 \mathrm{~nm}$ AlN films. The NC relays exhibit also a very high $I_{o n} / I_{\text {off }}$ ratio $\left(10^{8}\right)$ and an extremely small subthreshold slope $(0.015 \mathrm{mV} / \mathrm{dec})$, three orders of magnitude smaller than what is achieved by state-of-the-art MOSFETs.

The extremely small data dispersion in the measured $V_{t h}$ at a given $V_{B}$ for the demonstrated NC relays (Fig. 7b) indicates the weak influence of the contact's surface adhesion on the switching process (i.e. negligible hysteresis). Moreover, the precise control of $V_{\text {th }}$ in the range of few millivolts highlights the possibility of performing the switching function with just few millivolts. The NC piezoelectric relays demonstrated here have superior performance compared to the reported sub-1-Volt electrostatic relays [8] which exhibit unstable I-V characteristics and a high subthreshold slope 
of $10 \mathrm{mV} / \mathrm{dec}$. The NC relays have a relatively small actuator's footprint and an extremely small $V_{t h}$, which lead to a greatly reduced switching energy. For example, when $V_{G}=10 \mathrm{mV}$ (more than one order of magnitude larger than the minimum $V_{\text {th }}$ shown in Fig. 7a), the computed energy consumed per switching event is 46 aJ. This calculation assumes charging and discharging of an equal size gate. However, the active area of the NC relays is much larger than the active area of MOSFETs. A scaling analysis of AlN relays shows that the footprint of these devices could be reduced to 0.16 $\mu \mathrm{m}^{2}$ if $1 \mathrm{~nm}$ thick piezoelectric AlN films were to be used. In this case, the actuation voltage could be scaled to $41 \mathrm{mV}$.
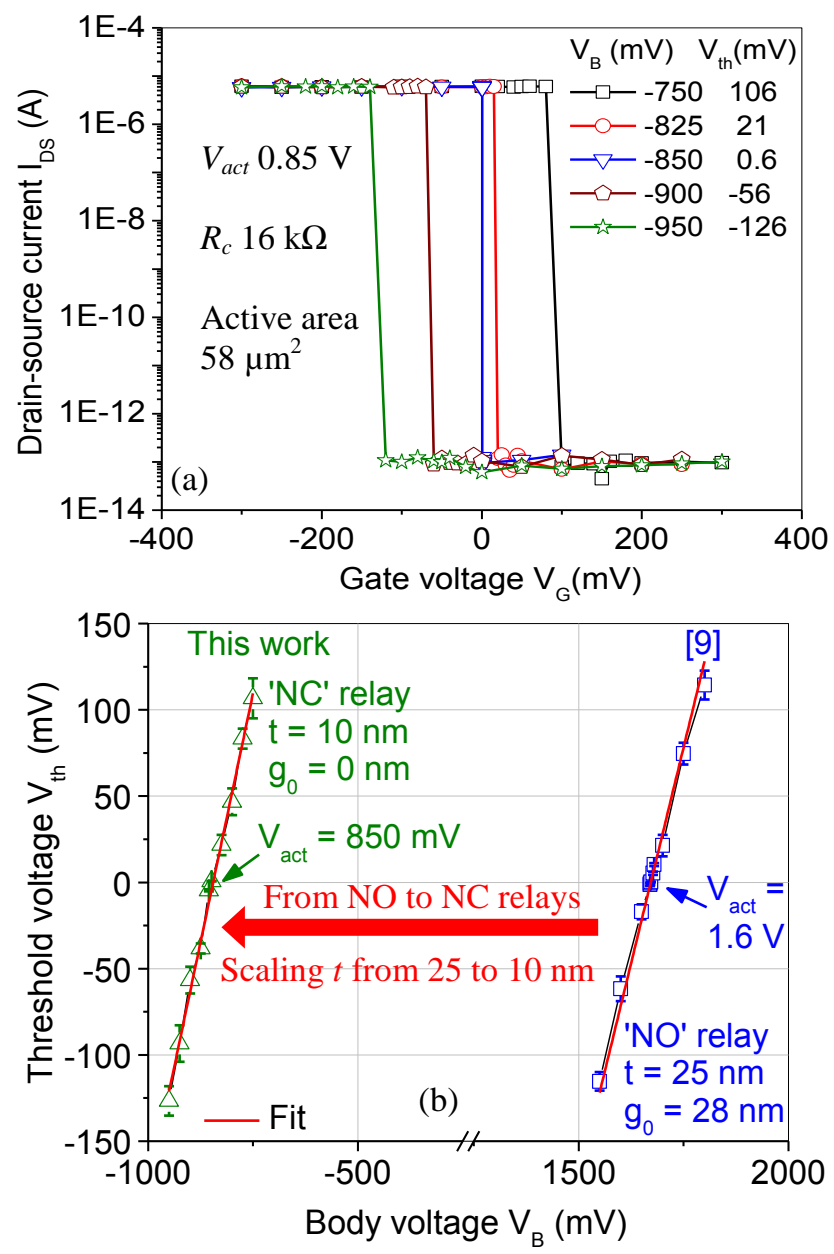

Figure 7: (a) The turn-off voltage sweeps of piezoelectric NC relays measured in ambient air using the body-biased method. The source-drain potential difference, $V_{D S}$, was $100 \mathrm{mV}$, and the minimum used $V_{G}$ step was $0.1 \mathrm{mV}$. (b) The average measured $V_{\text {th }}$ at different $V_{B}$ for the demonstrated $N C$ relays. The results of prior work on NO relays which use $25 \mathrm{~nm}$ AlN piezoelectric films is also plotted for comparison.

\section{CONCLUSION}

We demonstrated normally closed NEMS relays which use a stress compensating structure and $10 \mathrm{~nm}$ thick piezoelectric AlN films. The relays are the first to exhibit an actuation voltage smaller than $1 \mathrm{~V}$, and are capable of performing reliable switching with just few millivolts thanks to their extremely small subthreshold slope. The active area and actuation voltage of the demonstrated NC relays are respectively three and one orders of magnitude smaller compared to the normally open relays formed by $250 \mathrm{~nm}$ thick piezoelectric AlN films. These results clearly highlight the significant potential of scaling the AlN film thickness as well as employing the $\mathrm{NC}$ relay geometry. We believe this work has paved the way toward building ultralow-power microprocessors and low voltage memories.

Table 1: Summary of the measured data for the demonstrated NC relays and a comparison to prior work on AlN relays. The switches are referenced as S1, S2 and S3. Note that $S$ is the subthreshold slope and $R c$ is the source-drain resistance. The contact area of S1 is $2.4 \mu \mathrm{m}^{2}$.

\begin{tabular}{|l|c|c|c|}
\hline $\begin{array}{l}\text { Switch } \\
(\mathrm{NC} \text { or NO) }\end{array}$ & $\begin{array}{c}\text { S1 (NC) } \\
\text { This work }\end{array}$ & $\begin{array}{c}\text { S2 (NO) } \\
{[9]}\end{array}$ & $\begin{array}{c}\text { S3 (NO) } \\
{[11]}\end{array}$ \\
\hline$t / g_{0}(\mathrm{~nm})$ & $10 / 0$ & $25 / 28$ & $250 / 400$ \\
\hline$V_{\text {act }}(\mathrm{V})$ & $\mathbf{0 . 8 5}$ & $\mathbf{1 . 6 3}$ & $\mathbf{8 . 4 5}$ \\
\hline Active area $\left(\mu \mathrm{m}^{2}\right)$ & $\mathbf{5 8}$ & $\mathbf{1 8 7}$ & $\mathbf{4 0 0 0 0}$ \\
\hline$I_{\text {on }} / I_{\text {off }}$ & $10^{8}$ & $10^{7}$ & $10^{7}$ \\
\hline$S(\mathrm{mV} / \mathrm{dec})$ & $\mathbf{0 . 0 1 5}$ & 0.033 & 0.065 \\
\hline$R_{c}(\mathrm{k} \Omega)$ & 16 & 5.14 & 0.02 \\
\hline
\end{tabular}

\section{REFERENCES}

[1] O. Y. Loh and D. E. Horacio, "Nanoelectromechanical contact switches," Nature Nanotech., vol. 7, pp. 283-295, 2012.

[2] S. G. Narendra and A. Chandrakasan, "Leakage in Nanometer CMOS Technologies," Springer, New York, 2006.

[3] J. Jeon, V. Pott, H. Kam, R. Nathanael, E. Alon, and T. J. K. Liu, "Perfectly complementary relay design for digital logic applications," IEEE Electron. Dev. Lett., vol. 31, pp. 371-373, 2010.

[4] R. Proie, R. Polcawich, J. Pulskamp, T. Ivanov, and M. Zaghloul, "Development of a PZT MEMS switch architecture for low-power digital applications," J. of Microelectromech. Sys., vol. 20, pp. 1032-1042, 2011.

[5] K. Akarvardar and H. S. Wong, "Ultralow voltage crossbar nonvolatile memory based on energy-reversible NEM switches," IEEE Electron. Dev. Lett., vol. 30, pp. 626-628, 2009.

[6] W. Xiang and C. Lee, "Nanoelectromechanical torsion switch of low operation voltage for nonvolatile memory application," Appl. Phys. Lett., vol. 96, Art. \# 193113, 2010.

[7] T. Lee, S. Bhunia, and M. Mehregany, "Electromechanical computing at $500^{\circ} \mathrm{C}$ with silicon carbide," Science, vol. 329, pp. 1316-1318, 2010.

[8] J. O. Lee, Y. H. Song, M. W. Kim, M. H. Kang, J. S. Oh, H. H. Yang, and J. B. Yoon, "A sub-1-volt nanoelectromechanical switching device," Nature Nanotech., vol. 8, pp. 36-40, 2013.

[9] U. Zaghloul and G. Piazza, "10-25 nm piezoelectric nanoactuators and NEMS switches for millivolt computational logic," in Digest Tech. Papers MEMS'13 Conference, 2013, pp. 233-236.

[10] D. L. DeVoe and A. P. Pisano, "Modeling and optimal design of piezoelectric cantilever microactuators," $J$. Microelectromech. Syst., vol. 6, pp. 266-270, 1997.

[11] N. Sinha, T. Jones, Z. Guo, and G. Piazza, "Body-biased complementary logic implemented using AlN piezoelectric MEMS switches," J. of Microelectromech. Sys., vol. 21, pp. 484-496, 2012.

\section{CONTACT}

*G. Piazza: piazza@ece.cmu.edu

*U. Zaghloul: uzheiba@ andrew.cmu.edu 